\title{
Common Origin Tendon of the Biceps Femoris and Semitendinosus Muscles, Functional and Clinical Relevance
}

\author{
Tendón de Origen Común de los Músculos Bíceps Femoral \\ y Semitendinoso, Relevancia Funcional y Clínica
}

Emilio Farfán C. ${ }^{1}$; Marcia Gaete C. ${ }^{1}$; Ramón Olivé V. ${ }^{2}$ \& Alfonso Rodríguez-Baeza ${ }^{3}$

\begin{abstract}
FARFÁN, C. E.; GAETE, C. M.; OLIVÉ, V. R. \& RODRÍGUEZ-BAEZA, A. Common origin tendon of the biceps femoris and semitendinosus muscles functional and clinical relevance. Int. J. Morphol., 38(5):1341-1349, 2020.

SUMMARY: The injury of Biceps Femoris long head (BFlh) and Semitendinosus (ST) is caused by over-stretch of the muscles at the back of the thigh. This condition encompasses almost one third of sports-related injuries and has severe consequences, such as pain and weakness of the muscles. This injury is related namely to quick and demanding activities, such as short distance sprinting in a limited time period. The Common Origin Tendon (COT) for these two muscles is affected in these injuries. This tendon is formed by the BFlh and ST muscles which together have a common origin in the ischial tuberosity. Given the lack of complete knowledge about the detailed structure of the COT, we dissected cadaveric limbs, describing their morphological characteristics, and discussing its functional and clinical implications.: Thirty-five human cadaveric lower limbs were dissected after fixation to analyze the morphology of the COT, focusing on their volume and muscular/tendinous proportion. We identified two subtypes of COT. The most frequent was the musculotendinous type, in which the origin of the BFlh was mainly tendinous, while the ST muscle was mainly muscular (91.4\%). In the tendinous type both muscles had a tendinous origin (8.6\%). In the musculotendinous type, the ST muscle ends with a microscopic connective tissue that extend into the ischial tuberosity. We conclude that there is a variability in the anatomical presentations of the COT, and we propose that this will correspond with biomechanical differences in the risks and the response to regional injuries. The connective tissue between the COT and the neighbouring structures could be a risk factor for adhesion tearing.
\end{abstract}

KEY WORDS: Origin; Tendon; Bíceps Femoris; Semitendinosus.

\section{INTRODUCTION}

The injury of Biceps Femoris long head (BFlh) and Semitendinosus (ST) is caused by over-stretching of the muscles at the back of the thigh. These are common in sports that involve sudden increases and frequent changes in running speed, such as sprinting (Wangensteen et al., 2017), and can almost reach one-third of all sports-related injuries (Sivasundaram et al., 2015). Importantly, they and are the most common injury in professional soccer players (Ekstrand et al., 2011), representing $17 \%$ of the soccer players injuries from teams under the Union of European Football Associations (UEFA) and being the most common noncontact injury in elite football (van Dyk et al., 2018).

The Common Origin Tendon (COT) of the BFlh and
ST is a delicate structure located in the position where most of the muscle injuries are observed of the lower limb (Stepien et al., 2019); therefore, understanding the COT anatomy is crucial for therapeutic planification. These two muscles are connected by a $7.5 \mathrm{~cm}$ long aponeurosis extending from their sciatic origin (Standring, 2016), while their tendinous structure it is described to measure 5 to $6 \mathrm{~cm}$ in length (Paturet, 1951). The Semimembranosus (SM) muscle can be also considered in this common origin (Tubbs et al., 2016).

The origin of the COT is an oval area located $4 \mathrm{~cm}$ lateral to the vertex of the ischial tuberosity, with a surface area of approximately $10 \mathrm{~cm}^{2}$. The BFlh and the ST and SM muscles join in a common tendon approximately at $2 \mathrm{~cm}$ to

\footnotetext{
${ }^{1}$ Departamento de Anatomía, Escuela de Medicina, Pontificia Universidad Católica de Chile, Santiago, Chile. (ORCID: 0000-0002-8819-2945; ORCID: 0000-0003-1846-2417).

${ }^{2}$ Sports Medicine Department, Consorci Sanitari de Terrassa- CAR Sant Cugat, Barcelona, Spain, Facultad de Medicina y Ciencias de la Salud, Universitat Internacional de Catalunya, Barcelona, Spain. (ORCID: 0000-0001-6679-0437).

${ }^{3}$ Department of Morphological Sciences (Human Anatomy and Embryology Unit), Faculty of Medicine, Universitat Autònoma de Barcelona, Barcelona, Spain. (ORCID: 0000-0002-9181-5613).
} 
their ischial insertion (Neuschwander et al., 2015). The proximal insertion of these muscles is identified in two areas of the ischial tuberosity: an anterolateral insertion for the SM, and a posteromedial insertion for the BFlh and ST muscles. There is a direct connection between the tendon of the BFlh and the sacrotuberous ligament, which has not been observed in the case of the ST muscle (Sato et al., 2012). In these injuries, the pain is mainly located in the COT region (Lempainen et al., 2009). If some tearing is occurring in this area, it is important to elucidate if this tendinous region is formed mainly by tendon, muscle and/or connective tissue, indicative of a strong or weaker biomechanical properties to resist over-stretching or tearing events. Pain and adhesions will be also predictable for the presence of more connective tissue within the COT region, which is more prone to forming adhesions. Moreover, it is important to consider that the COT is adjacent to the sciatic nerve. This anatomical relation is clinically important as the sciatic nerve is often affected by injuries to the COT, which causes chronic neuropathy, and need to be considered in the differential diagnosis of the sciatic nerve disease (Bucknor et al., 2014). How COT and the sciatic nerve relation also need to be studied in detail.

In summary, information about the COT is focused on its functional, clinical and anatomical characteristics such us its relations and insertions (Sato et al.; Bucknor et al.; Neuschwander et al.; Tubbs et al.). However there are aspects of the COT that need to be reviewed: for instance, its morphology, structure, histology of the insertion in the ischial tuberosity and its anatomical relation with the sciatic nerve. Importantly, all the clinical implications of these aspects should also be discussed.

The aim of this study was to investigate the insertion of the COT in the ischial tuberosity and the composition of this structure in human lower limbs. We aimed to identify the tendinous, muscular and connective tissues present in this area all together with the anatomical relation with the sciatic nerve. The results of this work will help to understand the morphology of the COT and its functional and clinical implications: including the differential response to injury in this region, regarding vulnerability to tearing, adherence formation, pain and recovery.

\section{MATERIAL AND METHOD}

Preparation of the specimens. This study investigated 35 lower limbs from bodies donated to the Faculty of Medicine of the Universitat Autònoma de Barcelona. All the specimens were collected from persons that had expressed the willingness to donate their bodies for medical and scientific purposes signing a form approved by the Human Experimentation Ethics Committee of the Universitat Autònoma de Barcelona (procedure 2904 approved 27/03/ 2015). This 35 lower limbs from cadaveric human samples negative for hepatitis $\mathrm{B}$ and $\mathrm{C}$ and HIV, including just samples without signs of surgery in the region of interest. Bodies were preserved by arterial perfusion of Cambridge solution (phenol, ethanol, glycerin and formaldehyde) and maintained at $6{ }^{\circ} \mathrm{C}$ until their use. The average age of the subjects was $78.6 \pm 17.2$ years, $48.6 \%$ were male and 51.4 $\%$ female, while $57.1 \%$ of the lower limbs were right legs and $42.9 \%$ were left legs.

The anatomical dissection was conducted in planes. In the first step, the skin and subcutaneous cellular tissue were laterally lifted. The dissection started in the gluteal region until exposing its deep fascia. A descending section was made in the medial aspect of the thigh, extending horizontally to the lateral side of the limb. During this procedure, the fasciae latae muscle was preserved. In order to highlight the origin of the COT, the gluteal muscle in the proximal third of the thigh was sectioned and laterally removed, exposing the fascia that was covering the ischial tuberosity, as well as the COT, the sciatic nerve, the pelvic muscles and their associated vessels and nerves. Once the structures were exposed, the anatomical relations between the COT and the sciatic nerve were registered and investigated.

\section{Anatomical determinations were made as follows:}

Morphology of the COT: Fibres were classified as muscular, tendinous or both, based on their macroscopic observation.

Length of the COT: It was measured placing a tape in the midline of the COT insertion at the ischial tuberosity, which was considered as a proximal reference, and in the myotendinous junction of the BFlh.

Cross section diameter of the COT: The cross-section diameter of the COT was measured at three different levels: the tendon was divided into three equal portions. The thickness of the proximal part of the upper third, the central part of the middle third, and the distal end of the lower third.

Measurement of the tendinous area in comparison with the surrounding muscular tissue: One-centimetre thick cross sections of the COT were obtained at the three levels (upper third, middle third and lower third). The undersides where then photographed with a 12-Mpx camera on a tripod. The photographs were taken $26 \mathrm{~cm}$ above the sample. Areas of COT, the BFlh, ST and SM muscles were measured using ImageJ 1.51 software. 
Microscopic examination of the osteotendinous junction of common origin tendon: The structure of the proximal insertions of the ST muscle and the BFlh in the ischial tuberosity were analysed by histology. Whether the osteotendinous junction was muscular or tendinous was determined and registered for all the samples. For this, 10 randomly selected samples were fixed in formalin, decalcified and embedded in paraffin. Sections of $10 \mu \mathrm{m}$ were obtained and stained with Masson's trichrome.

Statistical analysis. Each measurement was done twice with different evaluators. Data obtained from these observations were analysed with SPSS 24.0 software. Ranges, frequencies, measures of central tendencies and standard deviations were calculated with a confidence interval of $95 \%$. The COT specimens were compared with a Tukey's multiple comparison test and ANOVA. In all cases, $\mathrm{p}<0.05$ was considered statistically significant and represented by asteriscs: ${ }^{*} \mathrm{p}<0.05 ; * * \mathrm{p}<0.01 ; * * * \mathrm{p}<0.001$.

\section{RESULTS}

The COT is formed by the proximal union of the tendon of the BFlh and the ST muscles, which reach their insertion in the ischial tuberosity, the tendon of the SM was observed how an independent tendon of the COT, being separable. In our sample, the composition of the COT was not the same in all cases, presenting variations in morphology, organization and anatomical relations.

The conformation of the COT was mainly musculotendinous in most of the samples. Two different conformations of the COT were observed, one consisting of a muscle-tendon (Fig. 1A) attachment and the other consisting of the attachment of two tendons (Fig. 1B).

In the muscle-tendinous conformation of the COT, the portion corresponding to the BFlh was a tendinous structure in all cases $(100 \%$, Fig. 1A, C). In contrast, the ST portion was a muscular structure in 32/35 of the cases (91.4 $\%$, Fig. 1B, C), and was attached to the ischial tuberosity throughout a short tendon located medially to the BFlh tendon (See arrow in Fig. 1A). In the other three cases (8.6 $\%$, all males), the ST muscle was completely tendinous in the COT area (Fig. 1B).

The muscle portion (ST) of the COT was inserted into the ischial tuberosity through a fibrocartilage and it represented most of its area. Histological microscopic analysis of the osteotendinous junction between the COT and the ischial tuberosity in the muscle-tendinous conformation showed that this region was formed by fibrocartilage of $1.148 \mathrm{~mm}( \pm 0.715)$ thick (Fig. 1D, E). The calcified portion of the fibrocartilage measured $0.542 \mathrm{~mm}$ $( \pm 0.435)$, while the uncalcified portion measured $0.606 \mathrm{~mm}$ $( \pm 0.354)$ (Fig. E). We detected similar proportions of the calcified vs uncalcified tissue (Fig. 1E), together with their histological similarities with the rest of the tissue external to them, that means, the uncalcified portion with the muscle and the calcified portion with the bone (Fig. 1D). This allows to infer that the COT has a transition tissue to transmit force to the ischial tuberosity, in which the non-calcified fibrocartilage could work together with the muscle, and the calcified fibrocartilage with the bony surface of the ischial tuberosity.

The morphology of the COT reveals an hourglass shape, while the semimembranous tendon is more prominent. While exposing the COT to perform its macroscopic observation, we observed that some loose connective tissue from the deep face of the gluteus maximus muscle, was arranged transversely, enveloping the COT, and was extended up to the ischial region. This loose tissue needed to be removed to allow the observation of the anatomy of the COT in detail.

The results of the macroscopic measurements of the COT were: mean length $67 \mathrm{~mm}$, and the mean cross section diameter of the upper third $25.5 \mathrm{~mm}$, medial third $21.7 \mathrm{~mm}$, and the lower third $26.8 \mathrm{~mm}$, presenting a hourglass shape (Fig. 2A,B Table I). Statistical analysis showed significant differences between the cross-section diameters of the three portions of the COT (Fig. 2B, One way ANOVA p< 0.001). Tukey multiple comparison tests showed that the average of the cross-section diameter of the middle portion was significantly smaller $(p<0.001)$ than those of the other two, which in turn did not show significant differences between them. Interestingly, in 28 of the cases $(80 \%)$, the mean diameter of the central cross-section was around $2 \mathrm{~mm}$ less than the diameters at the two thirds. In 3 cases, the mean diameter of the central cross-section was $1 \mathrm{~mm}$ less than that at the upper third, and in 4 cases $(11 \%)$, the mean diameter of the central cross-section was the same as those at the two thirds.

While most of these measurements formed a waist in two dimensions showing an hourglass shape, the area of the COT revealed an cone form: the average area of the upper third of the COT was $9.7 \mathrm{~mm}^{2}$, the area of the middle third was $16.5 \mathrm{~mm}^{2}$, and the total area of the lower third was 26.5 $\mathrm{mm}^{2}$ (see white columns in Fig. 2C). Measuring which muscle was represented in each third, the data show that the ST is the main component of the COT (Table I). In the case of the SM, the upper third is more represented (Fig. 2C, Table I). 


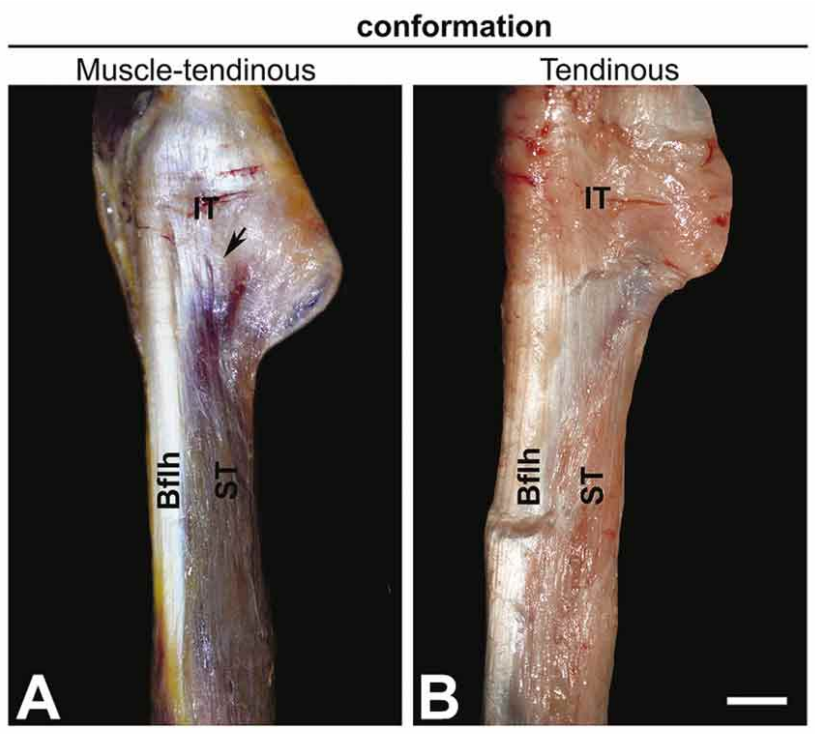

Histology at the bone insertion in the muscle-tendinous conformation

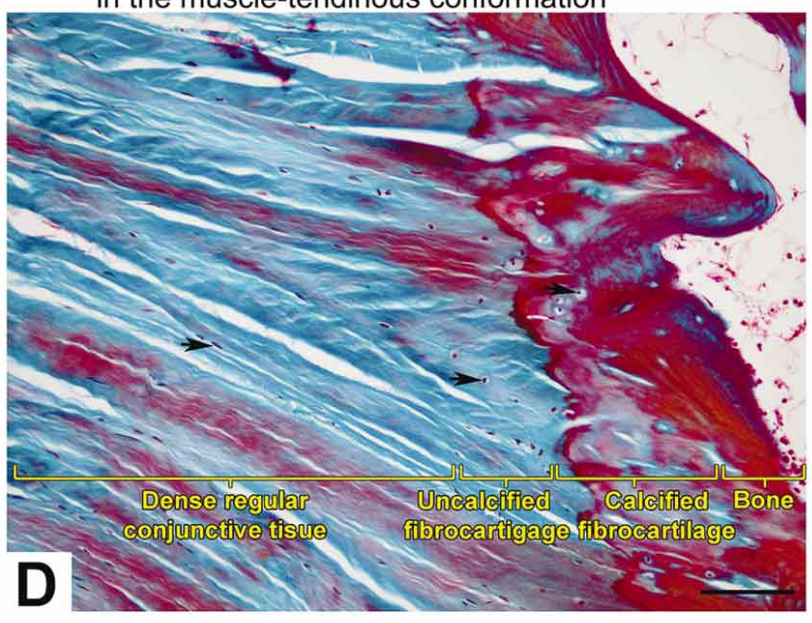

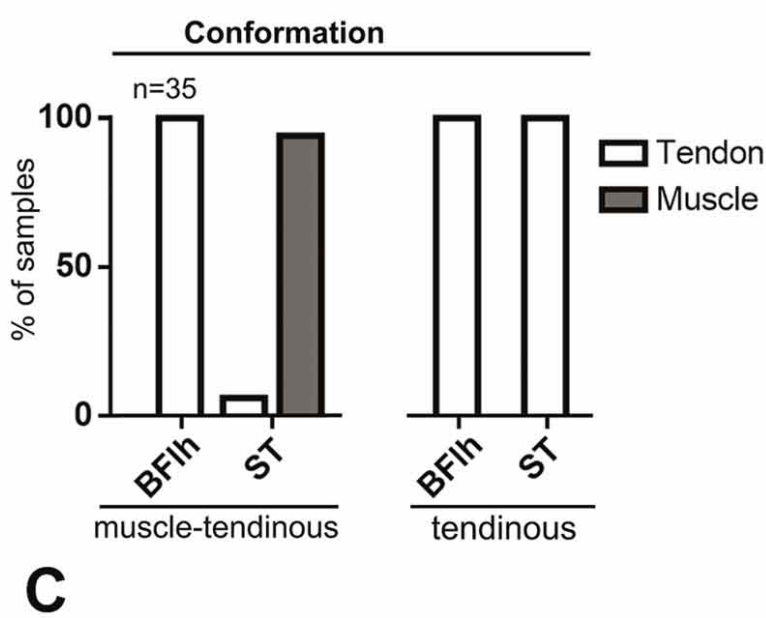

Fibrocartilage at the bone insertion in the muscle-tendinous conformation

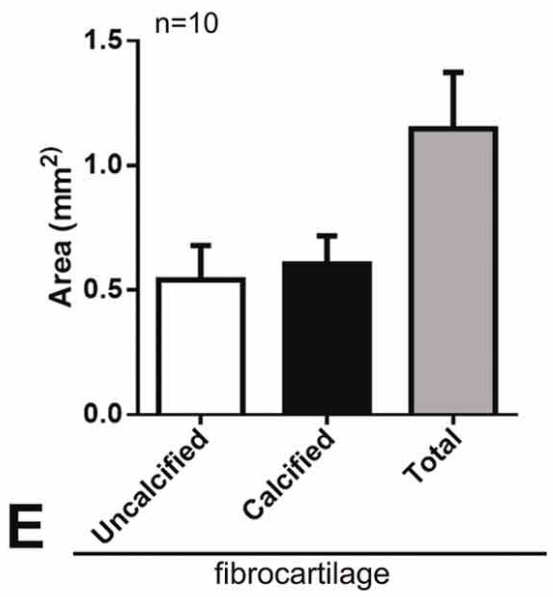

Fig. 1. Different conformations of the Common Origin Tendon: the musculo-tendinous and the tendinous conformations. (A-B) Posterior view of the Common Origin Tendon (COT). (A) Musculotendinous conformation. (B). Tendinous conformation. (C) Distribution of the muscles and tendons in both conformations. (D) Trichrome staining in histological longitudinal section at the level of the COT insertion in the ischial tuberosity (Masson's trichrome staining modified by Goldner), showing that a fibrocartilage form the connection between the muscle and bone in the musculotendinous conformation. In the dense regular conjunctive tissue of the tendon, the arrow indicates the fibroblast nucleus; in the uncalcified portion of the insertion fibrocartilage, the arrow indicates the chondrocyte nucleus; in the calcified portion of the insertion fibrocartilage, the arrow indicates the chondrocyte nucleus and finally the bone tissue comes from the ischial tuberosity. (E) Proportions of the fibrocartilage in this region. BFlh = Biceps Femoris long head muscle, ST = Semitendinosus muscle, IT = Ischial Tuberosity. Arrow $=$ Short tendon of the ST. Scale bars: A-B = 1cm; D = $100 \mu \mathrm{m}$.

The anatomical organization of the COT is variable and influences its anatomical relations. Regarding positioning of the structures that make up the COT, in 27 cases $(77.1 \%)$ the tendon of the BFlh muscle was located laterally to the tendon or muscular fibres of the ST muscle
(Fig. 3A). In 8 cases (22.9\%), the muscular fibres of ST origin were observed on the surface of the COT, covering the tendon of the BFlh (Fig. 3B). In these cases, the tendon of the BFlh was deeply embedded in the muscular fibres, but always positioned laterally, which could be verified by 

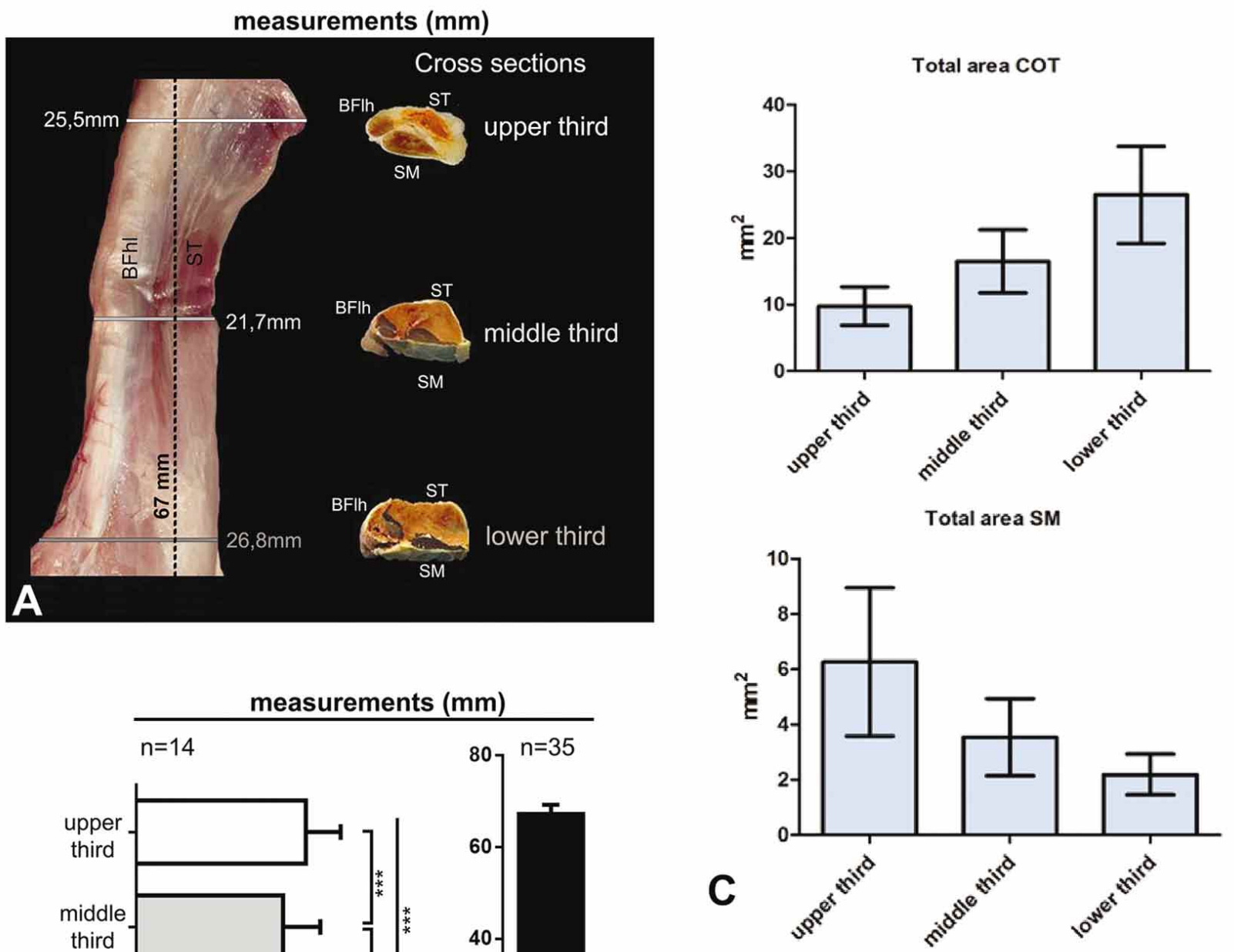

B

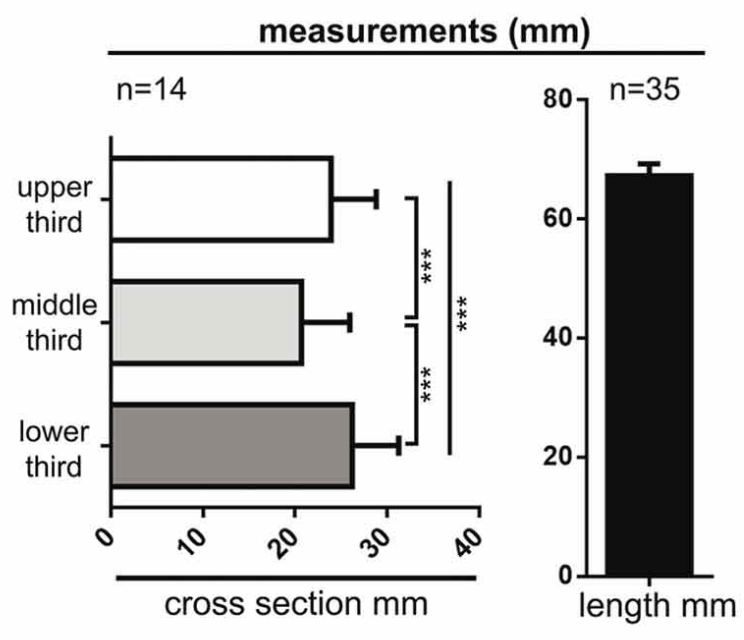

Fig. 2. The Common Origin Tendon has an hourglass shape. (A) Posterior view of the COT, the length and cross-section measurements and their respective lower view of three cross-section cuts of the COT at the levels of the upper, middle and lower third. (B) Graph of the quantifications represented in A, in which a hourglass shape is detected. (C) Muscular area of the region separated by muscles and the total area. The SM tendon area contributes more to the COT level compared to the ST and BFlh. BFlh = Biceps Femoris long head, ST $=$ Semitendinous.

observation and palpation. It was also confirmed that in these cases the union of the two muscles happened in the proximal third of the thigh, to a distance of $67 \mathrm{~mm}( \pm 12)$ from the ischial insertion (Fig. 1B). The cross-linking of the ST muscle fibres on the surface of the COT was determined, leaving the tendon of the BFlh anteriorly or laterally positioned in relation to ST muscle (Fig 3A, B). The posterior localization of the ST muscle in relation with the BFlh is atypical, due to normally the ST is medially located to the BFlh.
We investigated the anatomical relation between COT and the sciatic nerve. The sciatic nerve was located laterally to the COT, and covered by transversally arranged conjunctive tissue that comes from the fascia of the BFlh (Fig. 3A,B). This tissue was different from that coming from the gluteus maximus. This connection causes the displacement of the nerve when the COT is pulled. The nerve crosses deeply to the muscular belly of the BFlh, identifying connective tissue between these two structures. The COT 

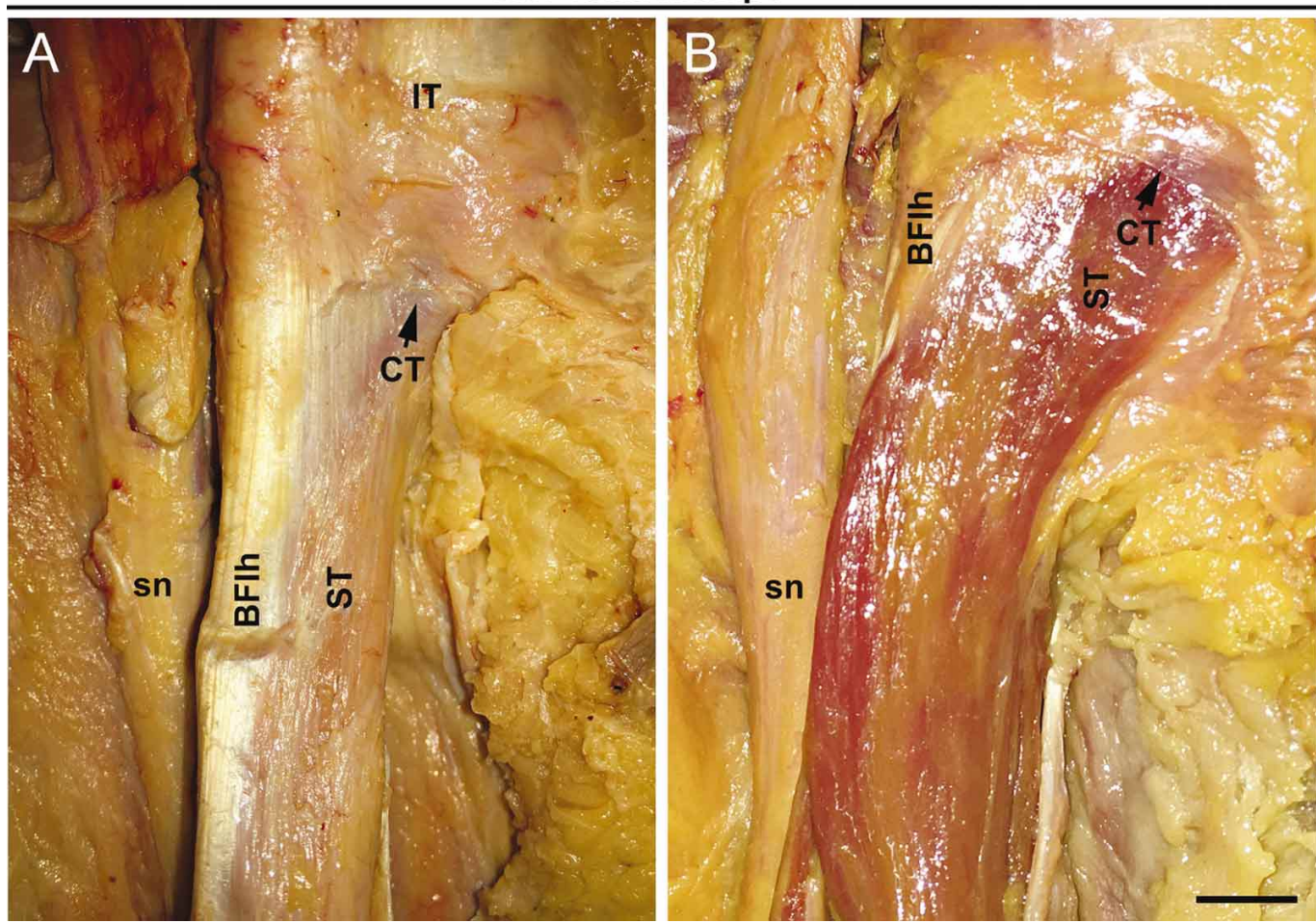

Fig. 3. The inferior third of the Common Origin Tendon is in relation with the sciatic nerve. Posterior view of the Common Origin Tendon, where two forms can be observed of the anatomical structural organization of the COT. (A) Shows the BFlh located laterally to the ST muscle. (B) Shows the BFlh, located anterior to the ST muscle. BFlh = Biceps Femoris long head muscle, $\mathrm{ST}=$ Semitendinosus, sn = sciatic nerve, $\mathrm{IT}=$ Ischial tuberosity covered by conjunctive tissue. $\mathrm{CT}=$ conjunctive tissue, which covers the COT. Scale bar $=1 \mathrm{~cm}$

Table I. Measurements of the common origin tendon of the biceps femoris and semitendinosus muscles.

\begin{tabular}{lcccc}
\hline Common tendon & Min. & Max. & Mean & SD \\
\hline Length $(\mathrm{mm})$ & 43 & 95 & 67 & 12 \\
Cross section diameter $(\mathrm{mm})$ & Min. & Max. & Mean & SD \\
Upper third cross section & 15 & 34 & 25.5 & 4.3 \\
Middle third cross section & 13 & 31 & 21.7 & 4.3 \\
Lower third cross section & 17 & 37 & 26.8 & 4.6 \\
ST area in the COT $\left(\mathrm{mm}^{2}\right)$ & Min. & Max. & Mean & SD \\
Upper third & 3.2 & 12.3 & 6.9 & 2.5 \\
Middle third & 5.3 & 23.6 & 12.8 & 4.1 \\
Lower third & 11 & 32.8 & 19.5 & 5.9 \\
BFlh area in the COT $\left(\mathrm{mm}^{2}\right)$ & Min. & Max. & Mean & SD \\
Upper third & 1.2 & 4.8 & 2.8 & 0.8 \\
Middle third & 1 & 6.9 & 3.7 & 1.4 \\
Lower third & 3.5 & 11.4 & 7 & 2.2 \\
Total area COT $\left(\mathrm{mm}^{2}\right)$ & ST & BFlh & Total & SD \\
Upper third & 6.9 & 2.8 & 9.7 & 2.9 \\
Middle third & 12.8 & 3.7 & 16.5 & 4.7 \\
Lower third & 19.5 & 7 & 26.5 & 7.3 \\
Total area SM $\left(\mathrm{mm}^{2}\right)$ & Min. & Max. & Mean & SD \\
Upper third & 2.5 & 12.7 & 6.3 & 2.7 \\
Middle third & 1.8 & 9.1 & 3.5 & 1.4 \\
Lower third & 0.8 & 3.6 & 2.2 & 0.7 \\
\hline
\end{tabular}

was also related to the gluteus maximus near its insertion in the ischial tuberosity interposing adipose tissue between both structures (see CT in Fig. 3A, B). In the medially localization of the ST in the COT, the sciatic nerve get close to a tendon (Figure 3A), while in the posterior localization of the ST in the COT is close to a muscle (Figure $3 \mathrm{~B}$ ).

\section{DISCUSSION}

The injuries of the BFlh and ST is the most common sports-related lower-limb muscular lesion, and the COT injuries is the most common non-contact injury in elite football (van Dyk et al.). It is particularly common among athletes that make forced hip flexion at the same time as extending the knee, as when sprinting, kicking or jumping. In many cases, the consequences of this injury implies the temporal cessation of the sport practice, and has a high rate of recidivism (12\% - 32\%) (Elliott et al., 2011). Prevention of this injury is achieved through a correct planning of the physical activity, which gradually allows these muscles to resist the demands of the sport. Consequently, anatomical 
knowledge of this muscular group is necessary to identify individual risks and develop preventive protocols, and, in case of injury, to support adequate treatment to avoid recurrence.

Few works have studied the anatomicalmorphological structure of the COT. Most of the research refers to the area of insertion of COT on the surface of the ischium or these clinical and fuctional importance. Moreover, this anatomical structure does not have an official term in the International Anatomical Terminology (Federative International Programme for Anatomical Terminology, 2019). When searching reliable databases such as "PubMed", hundreds of works appear under the concepts of "hamstring proximal", "hamstring proximal tendon", "origin of the hamstring tendon" etc. to refer to the common tendon of the BFlh and ST. There is no an anatomical term such as for example "conjoint tendon" that can include all these descriptions, which would facilitate the work of researchers, teachers and clinicians interested in this topic, being relegated to the anatomical term by descriptions that seek to explain something referred to this structure. For this reason, we believe it is necessary to give the COT an official anatomical term.

It is important to highlight that we discovered that this anatomical structure was normally musculotendinous, rather than solely tendinous. This has functional and clinical implications, due to the tendon and the muscle have different biomechanical characteristics: the tendon is designed to resist tension in one or multiple traction angles, while the contractile properties of the muscle allow its fibers to lengthen or shorten (Neumann, 2010). The highlighted difference can be detrimental to its tensile efficiency because of the difference in elongation coefficients between the muscle vs the tendon (Hamill et al., 2015). It has been done the biomechanical description of the stress and force curves of the tendons and ligaments, explaining that the initial tension of the tendon is followed by an elastic phase in which the tendon is able to progressively adapt to the applied load, maintaining a linear relation between load and stress. Thus, when the pulling force is removed, the tendon recovers its original length and shape, demonstrating that this elastic recovery contributes to mobilize the segment in movement (Oatis, 2009). In contrast, the mechanical response of the muscle is regulated by nervous stimulus (Enoka, 2015). For this reason, and from a biomechanical point of view, the behaviour of the COT differs depending whether it is tendinous or musculotendinous. When the conformation is tendinous, the COT's tension work is more efficient, with more competent levers and torques, whereas when the conformation is musculotendinous, a part of the tension work is replaced by contractile work, making it less efficient in mechanical terms. This is very important when considering the average areas measured at different levels of the COT. Overall, the ST muscle in all levels it had an area larger than the BFlh, represents $74.1 \%$ of the COT and the BFlh only $25.9 \%$ (average of three areas indicated in the Table I). It is also interesting to note that there was a proximal decrease of the area of the COT, inversely proportional to the increase of the area of the SM tendo (Fig. 2C, Table I), this could be interpreted as a way to compensate the muscular component of the ST, providing connective tissue to support the tensile requirements.

When the COT was tendinous, it was inserted directly in the ischial tuberosity on top of its fibers. In contrast, if the conformation was musculotendinous, the muscular fibres reached the tuberosity and were inserted through fibrocartilage that transmits the force between the bone and the muscle, and that during ossification of the ischial tuberosity may not have enough strength to support the traction of the COT (Fig. 1D). This observation could be related to avulsion injuries that occur with certain frequency among adolescents (Beltran et al., 2012) in which hyperflexion of the hip is combined with the extension of the knee (van der Made et al., 2019).

Also, we confirmed the presence of transversally arranged conjunctive tissue that covers the COT, which has been described, as a "retinaculum" that was considered a risk factor for tear by adhesion (Pérez-Bellmunt et al., 2015). This suggests that if the COT is musculotendinous, the risk of tearing by adhesion is greater than it is only tendinous, moreover in $22.9 \%$ of cases, ST muscle fibres covered the tendon surface, increasing the risk. When the ST muscle is superficial to the BFlh, the proximity of the gluteus maximus muscle to the COT is important. However, due to the amount of connective celluloid adipose tissue between them, adhesion injuries are less likely to occur. A COT tear tends to compromise the sciatic nerve with which it shares a conjunctive connection (Bucknor et al.), which give rise to neurological symptoms in $28 \%$ of cases (Wilson et al., 2017), causing denervation of the muscles innervated by the sciatic nerve (Aggen \& Reuteman, 2010), and alterations in sensitivity. Also, it has been described that the impairment in the sciatic nerve conductivity may exist in athletes with repeated stress injuries to this tendon (Kouzaki et al., 2017), which should be considered in athletes who have had muscular denervation as a consequence of a avulsion of the proximal tendon (Stepien et al.). This indicates that the anatomical relation between the COT and the sciatic nerve is clinically relevant, mainly at the level of the lower third of this tendon (Fig. 3) which as observed in this study, the COT has its largest cross section diameter, the contact surface with the sciatic nerve is greater and has been described as the most frequent site of injuries (van der Made et al., 2015). 


\section{CONCLUSIONS}

The anatomical structure of the Common Origin Tendon can vary, giving rise to different biomechanical behaviours in response to the tensions, which probably influence the formation of injuries. It is important to consider that the anatomical relation between COT and neighbouring structures, in particular, the sciatic nerve that can be injured. Knowledge about the morphology and functional characteristics of the COT will be useful for designing preventive and therapeutical strategies related to the COT injuries.

\section{ACKNOWLEDGEMENTS}

We thank the people that with great generosity donate their bodies to science, contributing to the development of new professionals and specialties.

FARFÁN, C. E.; GAETE, C. M.; OLIVÉ, V. R. \& RODRÍGUEZBAEZA, A. Tendón de origen común de los músculos bíceps femoral y semitendinoso, relevancia funcional y clínica. Int. J. Morphol., 38(5):1341-1349, 2020.

RESUMEN: Los desgarros de los músculos bíceps femoral cabeza larga (BFcl) y semitendinoso (ST) son causados por una tensión excesiva durante actividades que implican amplios rangos de movimiento en velocidad. Alcanzan casi a un tercio de las lesiones deportivas y tienen graves consecuencias competitivas, además generan dolor y debilidad muscular. El tendón de origen común (TOC) de estos dos músculos se ve afectado en estas lesiones. Este tendón se encuentra formado por el $\mathrm{BFcl}$ y el ST, originándose juntos en la tuberosidad isquiática. Debido a la falta de conocimiento completo y detallado de este tendón, se realizó un estudio cadavérico, describiendo sus características morfológicas y discutiendo sobre sus implicaciones funcionales y clínicas. Se practicó la disección anatómica de 35 miembros inferiores, lo que permitió describir la morfología del TOC, focalizando en su volumen y proporciones musculotendinosas. La conformación morfológica del TOC fue de 2 tipos: la musculotendinosa, donde el BFcl tenía una estructura tendinosa y el ST tenía una estructura muscular $(91,4 \%)$. La conformación tendinosa, donde ambos músculos tenían estructuración tendinosa $(8,6 \%)$. Cuando la conformación era musculotendinosa, el ST terminaba con un tejido conjuntivo microscópico, que se extendía hasta la tuberosidad isquiática. La conformación morfológica del TOC del BFcl y ST tuvo una presentación variable, lo cual podría tener implicación en su comportamiento biomecánico, y ser un factor de riesgo de lesiones. Además el tejido conjuntivo situado entre este tendón y las estructuras anatómicas vecinas podría ser un factor de riesgo de lesiones por adherencia.

PALABRAS CLAVE: Tendón; Origen; Bíceps femoral; Semitendinoso.

\section{REFERENCES}

Aggen, P. D. \& Reuteman, P. Conservative rehabilitation of sciatic nerve injury following hamstring tear. N. Am. J. Sports Phys. Ther, 5(3):143-54, 2010.

Beltran, L.; Ghazikhanian, V.; Padron, M. \& Beltran, J. The proximal hamstring muscle-tendon-bone unit: a review of the normal anatomy, biomechanics, and pathophysiology. Eur. J. Radiol., 81(12):3772-9, 2012.

Bucknor, M. D.; Steinbach, L. S.; Saloner, D. \& Chin, C. T. Magnetic resonance neurography evaluation of chronic extraspinal sciatica after remote proximal hamstring injury: a preliminary retrospective analysis. J. Neurosurg., 121(2):408-14, 2014.

Ekstrand, J.; Hägglund, M. \& Waldén, M. Epidemiology of muscle injuries in professional football (soccer). Am. J. Sports Med., 39(6):1226-32, 2011.

Elliott, M. C.; Zarins, B.; Powell, J. W. \& Kenyon, C. D. Hamstring muscle strains in professional football players: a 10-year review. Am. J. Sports Med., 39(4):843-50, 2011.

Enoka, R. Neuromechanics of Human Movement. $5^{\text {th }}$ ed. Champaign (I1.), Human Kinetics, 2015.

Federative International Programme for Anatomical Terminology (FIPAT). Terminologia Anatomica. Halifax, Dalhousie University, 2019. Disponible en: https://fipat.library.dal.ca

Hamill, J.; Knutzen, K. \& Derrick, T. Biomechanical Basis of Human Movement. $4^{\text {th }}$ ed. Philadelphia, Wolters Kluwer, 2015.

Kouzaki, K.; Nakazato, K.; Mizuno, M.; Yonechi, T.; Higo, Y.; Kubo, Y.; Kono, T. \& Hiranuma, K. Sciatic nerve conductivity is impaired by hamstring strain injuries. Int. J. Sports Med., 38(11):803-8, 2017.

Lempainen, L.; Sarimo, J.; Mattila, K.; Vaittinen, S. \& Orava, S. Proximal hamstring tendinopathy: results of surgical management and histopathologic findings. Am. J. Sports Med., 37(4):727-34, 2009.

Neumann, D. Kinesiology of the Musculoskeletal System. $2^{\text {nd }}$ ed. St. Louis, Mosby, 2010.

Neuschwander, T. B.; Benke, M. T. \& Gerhardt, M. B. Anatomic description of the origin of the proximal hamstring. Arthroscopy, 31(8):1518-21, 2015.

Oatis, C. Kinesiology. The Mechanics \& Pathomechanics of Human Movement. $2^{\text {nd }}$ ed. Baltimore, Lippincott Williams \& Wilkins, 2009.

Paturet, G. Traité d'Anatomie Humaine. Tome II. Membres Supérieur et Inférieur. París, Masson \& Cie éditeurs, 1951.

Pérez-Bellmunt, A.; Miguel-Pérez, M.; Brugué, M. B.; Cabús, J. B.; Casals, M.; Martinoli, C. \& Kuisma, R. An anatomical and histological study of the structures surrounding the proximal attachment of the hamstring muscles. Man. Ther., 20(3):445-50, 2015.

Sato, K.; Nimura, A.; Yamaguchi, K. \& Akita, K. Anatomical study of the proximal origin of hamstring muscles. J. Orthop. Sci., 17(5):614$8,2012$.

Sivasundaram, L.; Matcuk, G. R. Jr.; White, E. A.; Hatch, G. F. 3rd \& Patel, D. B. Partial semitendinosus tendon tear in a young athlete: a case report and review of the distal semitendinosus anatomy. Skeletal Radiol., 44(7):1051-6, 2015.

Standring, S. Gray's Anatomy. The Anatomical Basis of Clinical Practice. $41^{\mathrm{a}}$ ed. London, Elsevier, 2016.

Stepien, K.; Smigielski, R.; Mouton, C.; Ciszek, B.; Engelhardt, M. \& Seil, R. Anatomy of proximal attachment, course, and innervation of hamstring muscles: a pictorial essay. Knee Surg. Sports Traumatol. Arthrosc., 27(3):673-84, 2019.

Tubbs, R. S.; Shoja, M. M. \& Loukas, M. Bergman's Comprehensive Encyclopaedia of Human Anatomic Variation. New Jersey, Wiley Blackwell, 2016. 
van der Made, A. D.; Peters, R. W.; Verheul, C.; Maas, M. \& Kerkhoffs, G. M. Abduction in Proximal Hamstring Tendon Avulsion Injury Mechanism: A Report on 3 Athletes. Clin. J. Sport Med., 29(6):e76e79, 2019.

van der Made, A. D.; Wieldraaijer, T.; Kerkhoffs, G. M.; Kleipool, R. P.; Engebretsen, L.; van Dijk, C. N. \& Golanó, P. The hamstring muscle complex. Knee Surg. Sports Traumatol. Arthrosc., 23(7):2115-22, 2015.

van Dyk, N.; Bahr, R.; Burnett, A.F.; Verhagen, E.; von Tiggelen, D. \& Witvrouw, E. No association between rate of torque development and onset of muscle activity with increased risk of hamstring injury in elite football. Scand. J. Med. Sci. Sports, 28(10):2153-63, 2018.

Wangensteen, A.; Tol, J. L.; Roemer, F. W.; Bahr, R.; Dijkstra, H. P.; Crema, M. D.; Farooq, A. \& Guermazi, A. Intra- and interrater reliability of three different MRI grading and classification systems after acute hamstring injuries. Eur. J. Radiol., 89:182-90, 2017.

Wilson, T. J.; Spinner, R. J.; Mohan, R.; Gibbs, C. M. \& Krych, A. J. Sciatic nerve injury after proximal hamstring avulsion and repair. Orthop. J. Sports Med., 5(7):2325967117713685, 2017.
Corresponding author:

Prof. Emilio Farfán Cabello

Departamento de Anatomía

Escuela de Medicina

Pontificia Universidad Católica de Chile

Av. Libertador Bernardo O'Higgins \#340

Santiago

CHILE

E-mail: efarfanc@uc.cl

Received: 04-03-2020

Accepted: 30-04-2020 